R. J. Cohen and W. T. Sullivan, III, eds.

\title{
A Potential Site for the World's Largest Single Dish, FAST
}

\author{
B. Peng
}

Beijing Astronomical Observatory, National Astronomical

Observatories, Chinese Academy of Sciences, Beijing 100012, China

R. G. Strom

NFRA and University of Amsterdam, Postbus 2, 7990 AA Dwingeloo, The Netherlands

R. Nan

Beijing Astronomical Observatory, National Astronomical

Observatories, Chinese Academy of Sciences, Beijing 100012, China

\begin{abstract}
We have carried out a series of measurements at some locations in Guizhou Province and one additional site at the Urumqi Astronomical Station in the Xinjiang autonomous region, to check on their suitability, from the point of view of interference, for the construction of a Five-hundred-meter Aperture Spherical Telescope (FAST). This large facility will in some sense act as a prototype for the Square Kilometer Array (SKA). Measurements were made using a commercial receiver in the frequency range $25-1500 \mathrm{MHz}$. The results in Guizhou look quite promising. A protected radio quiet zone in Guizhou would make it an ideal location for an international radio astronomical facility, and would establish the FAST site as a natural SKA location.
\end{abstract}

\section{Introduction}

One way to realize the Square Kilometer Array (SKA), with a total collecting area of about $1 \mathrm{~km}^{2}$ to achieve $\simeq 1 \mu \mathrm{Jy}$ flux density sensitivity, is to construct a spherical reflector array of about 30 individual telescopes, each roughly $200 \mathrm{~m}$ diameter. Ideally, there would be continuous frequency coverage between about 0.2 and $2 \mathrm{GHz}$, with possible capability up to $5 \mathrm{GHz}$ or even $8 \mathrm{GHz}$ depending upon the cost. As the first step, a Five-hundred-meter Aperture Spherical Telescope (FAST) acting as a pilot for SKA has been proposed (Peng 1998).

FAST is not simply a copy of the existing Arecibo telescope, but has a number of innovations. Firstly, the proposed main spherical reflector (Qiu 1998), to fit a parabolid of revolution in real time by active control, enables the realization of broad bandwidth and full polarization capability by using standard feed design. Secondly, a feed support system that integrates optical, mechanical and electronic technologies (Duan et al. 1996) will effectively reduce the cost. With an overall diameter of $500 \mathrm{~m}$ and radius of its spherical surface of $300 \mathrm{~m}$, FAST will be the world's largest single dish. 


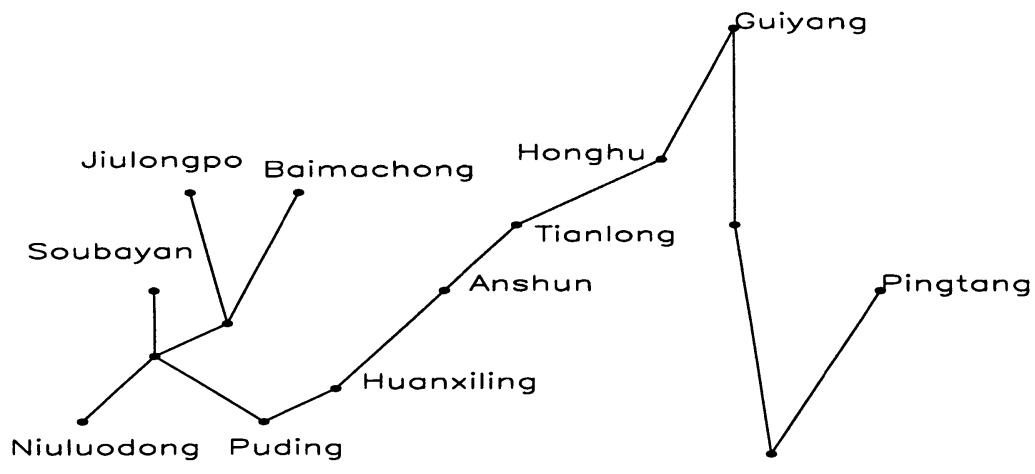

Figure 1. A schematic map of test sites measured in March 1995.

There are a number of important considerations affecting the choice of any telescope site. It has been realized that the right terrain, such as the Karst formations of Guizhou Province in southwestern China, would be critical to a successful implementation of an Arecibo-style spherical reflector (Braun 1994). The Chinese FAST/KARST ${ }^{1}$ project team has been searching for suitable sites and testing them for an Arecibo-style telescope. A database of about 400 Karst depressions in Pingtang and Puding counties in the province has been set up. It is well-known that a critical consideration in choosing the best telescope site is the interference environment. As part of the international effort to build such a new telescope, the Chinese Academy of Sciences and the Royal Dutch Academy of Sciences have funded a bilateral exchange program to enable radio interference measurements to be carried out at a number of potential sites in China. In the following, we present preliminary results from a series of interference monitoring tests at various locations in Guizhou and Xinjiang.

\section{Preliminary Results}

In November 1994, the first measurements were made at 8 Karst depression sites located at an altitude of about $1,000 \mathrm{~m}$ above sea level in Pingtang and Puding counties in Guizhou. In addition, we monitored a site in the centre of Guiyang, the capital of Guizhou, and as a further test, made measurements at the site of the $25 \mathrm{~m}$ telescope of the Urumqi Astronomical Station in the Xinjiang autonomous region in northwestern China. Guiyang was monitored just to compare the interference situation in a populated area with that of the remote sites. Four months later in March 1995, additional observations were made at a number of remote sites for comparison. These included monitoring at four Karst depressions in Puding for a period of one month in order to obtain

\footnotetext{
${ }^{1}$ Kilometer-square Area Radio Synthesis Telescope, a Chinese concept for the SKA project.
} 

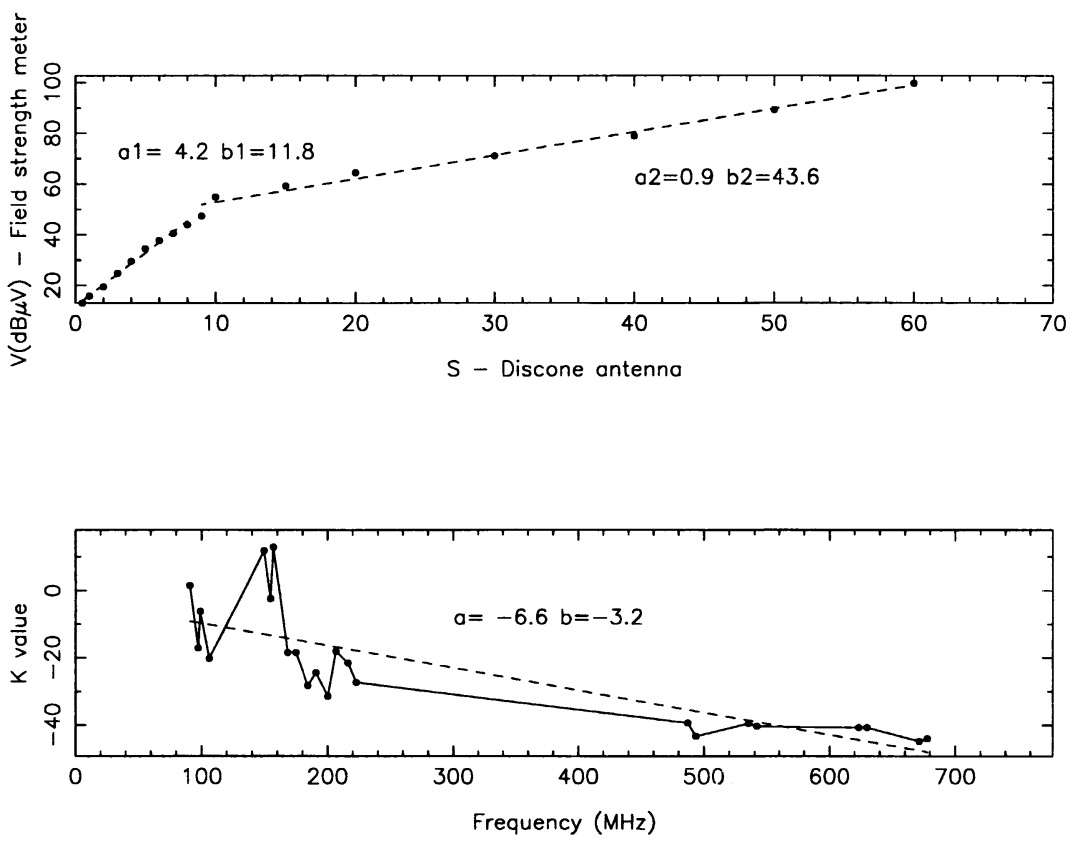

Figure 2. Upper plot: Relative voltage $\mathrm{S}$ versus real voltage $V_{r} ;$ Lower plot: $K$ coefficient of discone antenna versus frequency.

more complete statistics; and an investigation of interference at several locations between sites in Puding county, Anshun and Guiyang cities, and Pingtang county in Guizhou in an attempt to understand distance effects. A schematic route of this trip, not shown to scale, is plotted in Figure 1

The measurements were carried out with an Icom IC-R7100 receiver coupled to a Diamond Antenna Corp. D-130N omnidirectional discone antenna. This combination can receive over the range $25-1500 \mathrm{MHz}$ (the receiver is continuously tunable up to $2 \mathrm{GHz}$, and a check showed that the antenna can receive there, but presumably with reduced gain). The $\mathrm{R} 7100$ can receive and detect single side band (SSB), both upper and lower side bands (USB and LSB), and $\mathrm{AM}$ and FM (in wide and narrow-band mode) signals. For the purpose of our observations, we scanned in SSB (USB) mode, but most signals found were then checked in the various modes, particularly to look for voiced modulation. The measurements were done in automatic scanning mode, with the squelch threshhold set just above the background noise level (about 1 on the S-meter) so that any signal above noise would be detected. A few scans were made by hand to search for signals in the noise, and a number were found. The scan step was usually $10 \mathrm{kHz}$ below $200 \mathrm{MHz}$, and $25 \mathrm{kHz}$ above. About $2.5 \mathrm{hr}$ was typically required to monitor each site over the entire $25-1500 \mathrm{MHz}$ range.

To put our values on an absolute intensity scale, we calibrated both the receiver and the antenna. The field measurements of intensity give the relative voltage $(S)$ of signals picked up at each interfering frequency. Using a signal 

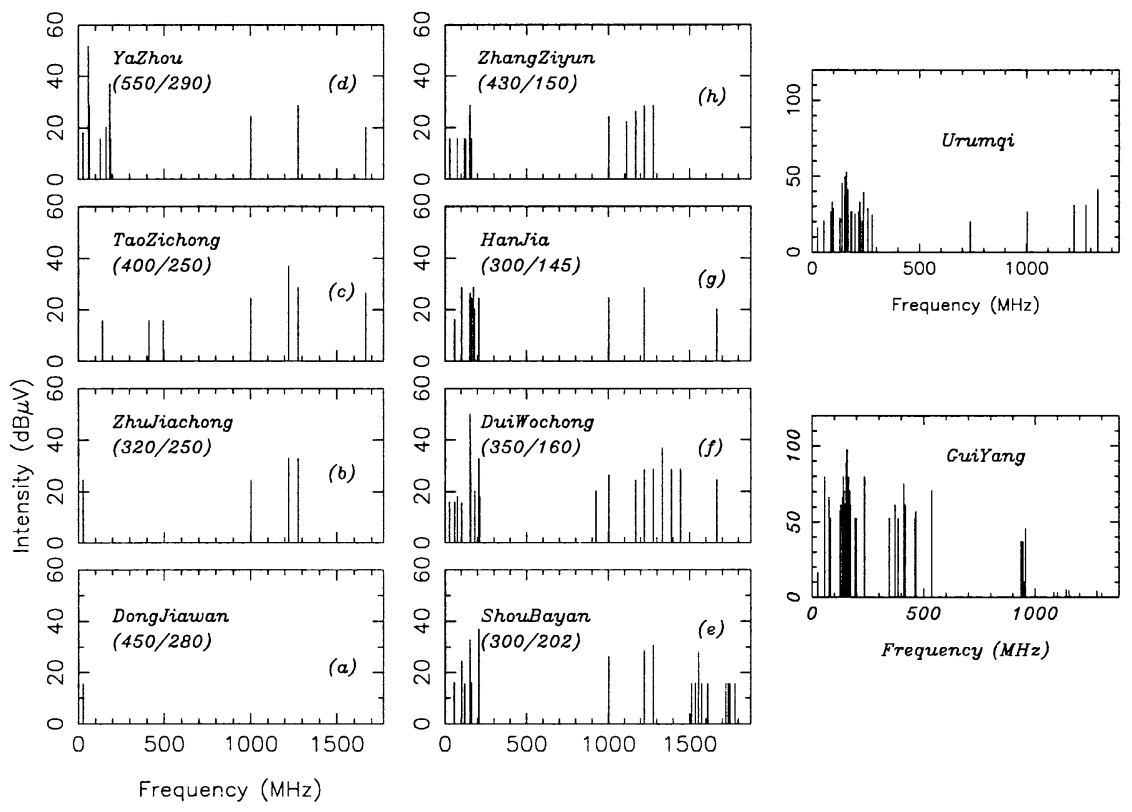

Figure 3. The first measurements of interference levels $E$, made in November 1994.

generator (MG615B) we have determined $S$ and the real voltage $V_{r}$ at frequencies of $30,100,200,400$ and $800 \mathrm{MHz}$. The measured $S$ (of the IC-R7100) versus $V_{r}$ $(\mathrm{dB} \mu \mathrm{V})$ (of the ML518 field strength meter), based on average values of $V_{r}$, is shown in Figure $2(0 \mathrm{~dB} \mu \mathrm{V}=-60 \mathrm{dBmV})$, the upper plot. By least-squares fit we have, for $S \leq 9.0$,

$$
V_{r}(\mathrm{~dB} \mu \mathrm{V})=4.2 \times S+11.8
$$

otherwise,

$$
V_{r}(\mathrm{~dB} \mu \mathrm{V})=0.9 \times S+43.6
$$

The $K$ coefficient of the D-130N omnidirectional discone antenna, defined as $V_{r}-E$ (the field strength), can be approximated by

$$
K=-6.6 \times f(\mathrm{MHz})-3.2,
$$

and is plotted in Figure 2 at the bottom. It has been measured over a frequency range of $50 \mathrm{MHz} \sim 700 \mathrm{MHz}$ using a log-periodic antenna MP635A coupled to the ML518 field strength meter. This gives us a general idea of the absolute interference strength $E$ at the surveyed sites.

The results of our measurements are presented in Figures 3, 4 and 5. In the left 8 graphs of Figure 3, the name of each Karst depression is labelled at the top left: the diameter $D$ and the depth $H$ were roughly estimated (in meters) and are given just under each name in the form $D / H$. Four depressions, 

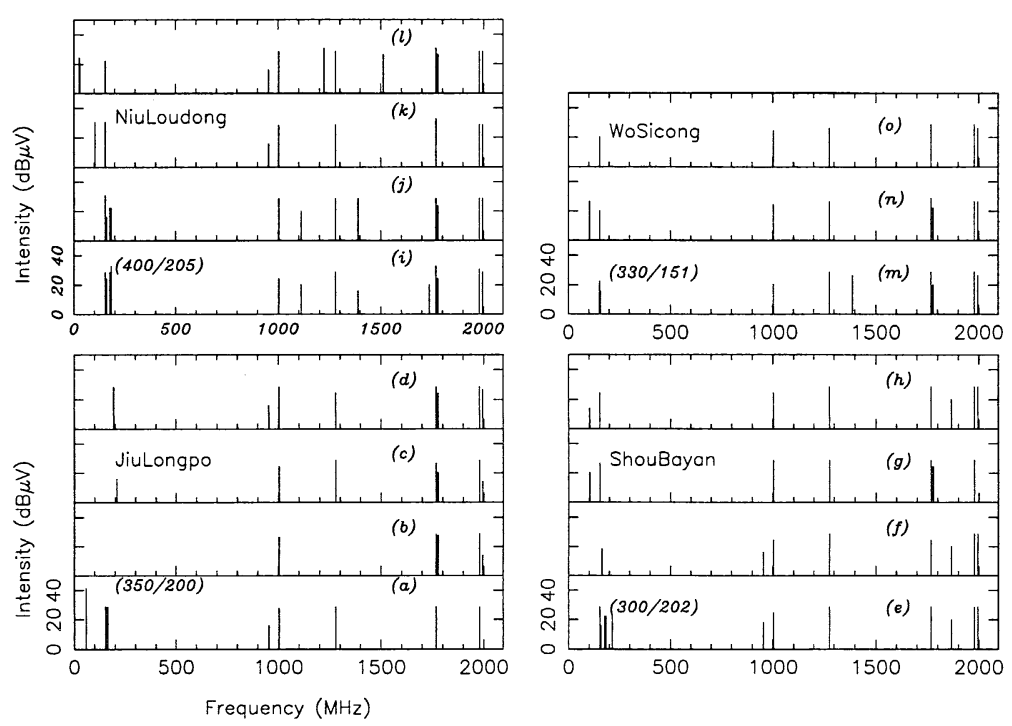

Figure 4. Weekly monitoring at four locations in Puding county, March 1995.

$\mathrm{a}, \mathrm{b}, \mathrm{c}$ and $\mathrm{d}$, lie in Pingtang county, and the others in Puding county. Some signals at frequencies of $256,512,768,1024,1500 \mathrm{MHz}$ and their harmonics were found to be produced by the quartz oscillators of the receiver itself; they have not been reproduced in the figures. The lower right plot in Figure 3 shows the interference situation in Guiyang city, which was measured from the top of an 11-storey building of the Science and Technology Commision of Guizhou Province, the highest structure in that part of the city. The upper right plot shows the situation at the $25 \mathrm{~m}$ telescope station, about $50 \mathrm{~km}$ southwest of Urumqi city, the capital of Xinjiang.

Figure 4 contains 15 graphs, showing the interference monitoring results for four Karst depression sites during one month in Puding, with an interval of one week between measurements at each location. It seems that the interference environment there was quite stable during this period. Results obtained in March 1995 are presented in Figure 5 by means of graphs that show interference measurements of eleven sites ordered according to their geographic location (see Fig. 1). These include four Karst depressions in Puding and sites going from Puding via Anshun and Guiyang to Pingtang. They give us some indication of variations with distance. As one can clearly see, at frequencies below $1000 \mathrm{MHz}$ a few sources of radio interference are strong, and some wide band interference is also present in Guiyang City. 

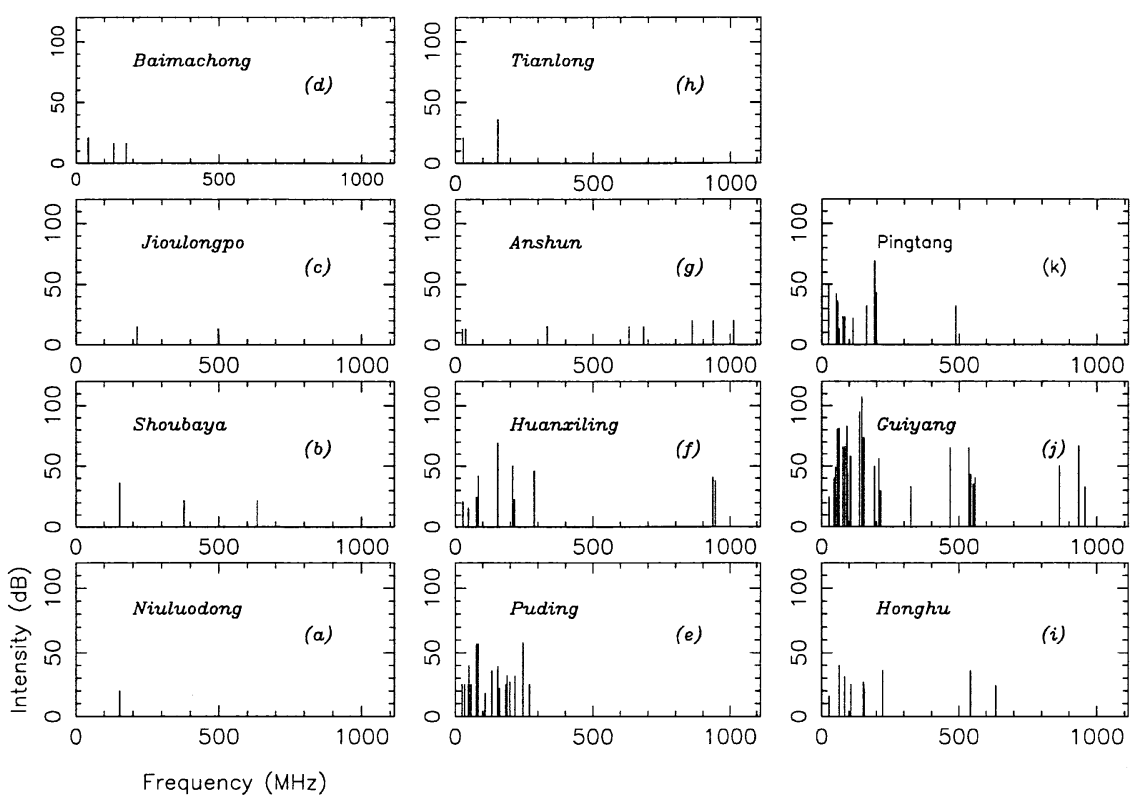

Figure 5. Interference measurements ordered by geographic location (see Fig. 1), March 1995.

\section{Concluding Remarks}

The results of these measurements provide information about the frequency, strength and characteristics of the interfering signals. Most of the interfering signals found appear to be narrow-band $(<10 \mathrm{kHz})$ beacon signals of unknown origin. There are also a few FM radio stations, mobile communication bands and television channels. Moreover, additional observations were made at remote sites for comparison, a longer period of one month's monitoring at four sites in Puding for more complete statistics, and an investigation of interference at several locations between sites from Puding via Anshun and Guiyang cities to Pingtang in Guizhou to understand distance effects.

Qualitatively and semi-quantitatively, the data are about what one might expect. As one leaves Guiyang (Fig. 5j) going west, the interference level generally and number of interfering signals drops in Honghu (Fig. 5i). Continuing to Tianlong (Fig. 5h) it decreases further, but as one approaches Anshun (Fig. 5g, which was outside of the city itself) the numbers increase again. As for changes with time, Fig. 4 shows that the situation at four sites was quite stable over a month. For one of these, Shou Bayan (Fig. $4 \mathrm{e}-\mathrm{h}$ ), the picture is quite similar to that four months earlier (Fig. 3e).

All in all, we conclude that, due to the remoteness of this region and terrain shielding, the results in Guizhou Province look quite promising, with relatively little interference found in the region $220-920 \mathrm{MHz}$. 
A State Standard, "Protection Criterion for the Radio Astronomy Service" has been submitted to the State Supervisory Bureau of Technologies, and is expected to be issued as one of the laws for radio management in China. Recently we made proposals for designating a radio quiet zone (RQZ) in Guizhou province for a potential site of FAST.

Guizhou regional Bureau of Radio Management agreed to work and collaborate on designating a RQZ as soon as the final site for FAST/KARST was chosen and the project FAST approved as a National Megascience Project of China. Guizhou provincial government would like to make a plan to modestly develop their radio industry and communications. We have resumed monitoring the radio interference environment since January 1999, and believe that a protected RQZ in Guizhou would make it an ideal location for an international radio astronomical facility, and would establish the FAST site as a natural SKA location.

Acknowledgments. B. Peng thanks the MPIfR in Germany and the IAU travel grants for supporting his attendance at this IAU symposium 196. We are grateful to the Chinese Academy of Sciences and the Royal Netherlands Academy of Sciences for travel grants in the context of a bilateral exchange programme (grant number 96CDP042) which made much of this research possible. We wish to thank all the members of the FAST/KARST project team for their efforts in such a site survey, the S\&T Commission of Guizhou Province, the Institute of Remote Sensing Application, the officials of counties Pingtang and Puding, and the Karst Comprehensive Research and Experimental Station for their practical help. This FAST project has now become a key project in the Chinese Academy of Sciences, and has also been supported by the Ministry of Science and Technology of China.

\section{References}

Braun, R. 1994, Draft Minutes of URSI LTWG-1-minutes, Jodrell Bank

Qiu Y.H. 1998, MNRAS, 301, 827-830

Peng B. 1998, The Observatory, 118, p.261 